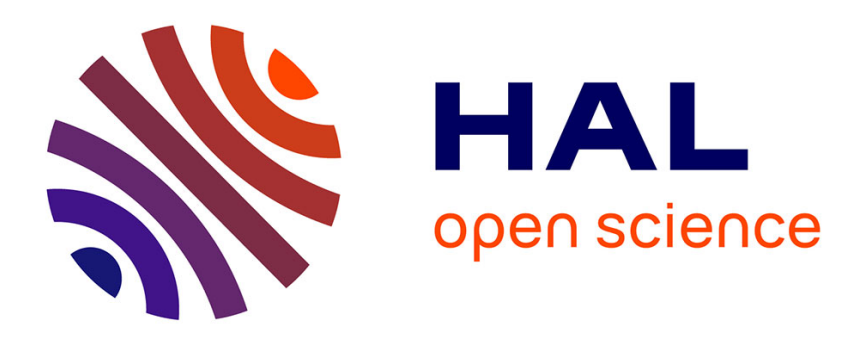

\title{
Hyperbolic approximation of the BBM equation
}

Sergey Gavrilyuk, Keh-Ming Shyue

\section{To cite this version:}

Sergey Gavrilyuk, Keh-Ming Shyue. Hyperbolic approximation of the BBM equation. 2021. hal03255398

\section{HAL Id: hal-03255398 \\ https://hal.science/hal-03255398}

Preprint submitted on 9 Jun 2021

HAL is a multi-disciplinary open access archive for the deposit and dissemination of scientific research documents, whether they are published or not. The documents may come from teaching and research institutions in France or abroad, or from public or private research centers.
L'archive ouverte pluridisciplinaire HAL, est destinée au dépôt et à la diffusion de documents scientifiques de niveau recherche, publiés ou non, émanant des établissements d'enseignement et de recherche français ou étrangers, des laboratoires publics ou privés. 


\title{
Hyperbolic approximation of the BBM equation
}

\author{
Sergey Gavrilyuk; Keh-Ming Shyue ${ }^{\dagger}$
}

June 9, 2021

\begin{abstract}
It is well known that the Benjamin-Bona-Mahony (BBM) equation can be seen as the Euler-Lagrange equation for a Lagrangian expressed in terms of the solution potential. We approximate the Lagrangian by a oneparmeter family of Lagrangians depending on three potentials. The corresponding Euler-Lagrange equations can be then written as a hyperbolic system of conservations laws. The hyperbolic BBM system has two genuinely non-linear eigenfields and one linear degenerate eigenfield. Moreover, it can be written in terms of Riemann invariants. Such an approach conserves the variational structure of the BBM equation and does not introduce the dissipation into the governing equations as it usually happens for the classical relaxation methods. The state-of-the-art numerical methods for hyperbolic conservation laws such as the Godunov-type methods are used for solving the 'hyperbolized' dispersive equations. We find good agreement between the corresponding solutions for the BBM equation and for its hyperbolic counterpart.
\end{abstract}

Keywords : nonlinear dispersive equations, hyperbolic equations, EulerLagrange equations

Mathematics Subject Classification numbers: 35L65, 35Q35.

\section{Introduction}

The Benjamin-Bona-Mahony (BBM) equation was proposed as a unidirectional model of weakly non-linear waves in shallow water [5]. It can be written in the following form :

$$
u_{t}+u u_{x}-u_{t x x}=0 .
$$

The dispersion relation to (1) linearized on a constant solution $u=u_{0}$ is

$$
c_{p}=\frac{u_{0}}{1+k^{2}},
$$

\footnotetext{
*Aix Marseille Univ, CNRS, IUSTI, UMR 7343, Marseille, France, sergey.gavrilyuk@univamu.fr

${ }^{\dagger}$ Institute of Applied Mathematical Sciences, National Taiwan University, Taipei 106, Taiwan, shyue@ntu.edu.tw
} 
where $c_{p}$ is the phase velocity, and $k$ is the wave number. It follows from (2) that the phase velocity and group velocity are bounded for any wave number, i.e. the BBM equation has in some sense hyperbolic properties.

The idea of this work is to approximate the BBM equation by an 'equivalent' hyperbolic system such that the solutions of the hyperbolic system are close to the solutions of the BBM equation. Recently, there has been an increasing interest in rewriting nonlinear partial differential equation (PDE) systems with higher order derivatives under the form of nonlinear hyperbolic relaxation systems, in conjunction with potentially stiff algebraic relaxation source terms. The idea goes back to the seminal work of Cattaneo [10], who proposed a hyperbolic reformulation of the parabolic heat equation. More recent work on the topic also regards the hyperbolic relaxation reformulation of dissipative continuum mechanics $[16,17]$ as well as the hyperbolic reformulation of nonlinear dispersive systems $[40,1,11,4,43]$.

The relaxation technique is commonly associated with dissipative process. The question appears if such a technique could be applied for conservative dispersive systems which are the Euler-Lagrange equations for a 'master' Lagrangian. Such a 'hyperbolic' approach was recently proposed in [23, 9] for the Serre-Green-Naghdi (SGN) model [44, 26, 27] describing long dispersive waves, for the nonlinear defocusing Schrödinger equation [14], and for a bistable Ericksen bar [8]. The PDE systems were derived from an 'augmented' ('extended') Lagrangian and have a rather similar mathematical structure. The corresponding 'augmented' Lagrangian approximating a given 'master' Lagrangian represents a parametric family of Lagrangians depending on a larger set of variables.

The advantages of such a conservative hyperbolic formulation are multiple. First, from Noether's theorem one recovers the analogues of conservation laws admitted by the Euler-Lagrange equations for the 'master' Lagrangian, i.e. the physical meaning of conservation laws is not lost. Second, no need to invent new numerical methods which are specific to a given dispersive equation : the state-of-the-art numerical methods for hyperbolic conservation laws such as the Godunov-type methods can be used for solving the 'hyperbolized' dispersive equations. Third, and not least, the boundary conditions are easier to treat for the hyperbolic equations than for the dispersive equations where such a treatment is specific to the equations to study : [6] (linear Korteweg-de-Vries equation), [2, 3] (linear and nonlinear Schrödinger equation), [7] (linear BBM equation), [32] (linear Serre-Green-Naghdi equations), [35] (Boussinesq system).

The construction of the extended Lagrangian is intuitive rather than rational. We have only to make sure that the solutions of the extended hyperbolic system are close to the solutions of the original system. The comparison between numerical and analytical solutions of the exact and approximate equations becomes thus crucial. Sometimes, a rigorous proof of this fact can be made. This is the case, for example, of the SGN equations [15]. 
The 'master' Lagrangian for the BBM equation (1) can be written as

$$
\mathcal{L}=-\frac{\varphi_{t} \varphi_{x}}{2}+\frac{\varphi_{t} \varphi_{x x x}}{2}-\frac{\varphi_{x}^{3}}{6}, \quad u=\varphi_{x}
$$

Indeed, the Euler-Lagrange equation for (3) is :

$$
-\frac{\partial}{\partial t}\left(\frac{\partial \mathcal{L}}{\partial \varphi_{t}}\right)-\frac{\partial}{\partial x}\left(\frac{\partial \mathcal{L}}{\partial \varphi_{x}}\right)-\frac{\partial^{3}}{\partial x^{3}}\left(\frac{\partial \mathcal{L}}{\partial \varphi_{x x x}}\right)=\varphi_{t x}+\varphi_{x} \varphi_{x x}-\varphi_{x x x t}=0 .
$$

The function $\varphi$ is thus a potential for the variable $u$. In [41] it is justified that (1) admits only three independent conservation laws :

$$
\begin{gathered}
\left(u-u_{x x}\right)_{t}+\left(\frac{u^{2}}{2}\right)_{x}=0 \\
\left(\frac{u^{2}}{2}+\frac{u_{x}^{2}}{2}\right)_{t}+\left(\frac{u^{3}}{3}-u u_{t x}\right)_{x}=0 \\
\left(\frac{u^{3}}{3}\right)_{t}-\left(u_{t}^{2}-u_{x t}^{2}+u^{2} u_{x t}-\frac{u^{4}}{4}\right)_{x}=0 .
\end{gathered}
$$

A Hamiltonian formulation of the BBM equation can be found in [42].

We propose to replace the BBM equation by a hyperbolic system of equations obtained as the Euler-Lagrange equations for an 'augmented' Lagrangian. In particular, we will show that the dispersion relation for the BBM equation and that coming from the approximate system are close to each other. Moreover, we will prove that the approximate hyperbolic model can be written in terms of the Riemann invariants. Finally, we find good agreement between the numerical solutions to both models.

\section{Augmented Lagrangian}

Let us introduce three potentials $\varphi, \psi$ and $\chi$ and the corresponding variables $u, v, w$ defined as $u=\varphi_{x}, v=\psi_{x}$ and $w=\chi_{x}$. Let us define a one-parameter family of 'augmented' Lagrangians :

$$
\hat{\mathcal{L}}=-\frac{\varphi_{t} \varphi_{x}}{2}-\frac{\varphi_{x}^{3}}{6}-\frac{\psi_{t} \psi_{x}}{2 c}-\varphi_{x} \psi_{x}+\psi_{x} \chi-\frac{\chi_{t} \chi_{x}}{2}-\frac{\chi_{x}^{2}}{2 c} .
$$

Here $c>0$ is a large positive constant. Then the Euler-Lagrange equations for (7) can be written as :

$$
u_{t}+u u_{x}+v_{x}=0, \quad \frac{v_{t}}{c}+u_{x}=w, \quad w_{t}+\frac{w_{x}}{c}=-v .
$$


Indeed, the variational derivatives with respect to $\varphi, \psi$ and $\chi$ should vanish :

$$
\begin{aligned}
& \frac{\delta \hat{\mathcal{L}}}{\delta \varphi}=-\frac{\partial}{\partial t}\left(\frac{\partial \hat{\mathcal{L}}}{\partial \varphi_{t}}\right)-\frac{\partial}{\partial x}\left(\frac{\partial \hat{\mathcal{L}}}{\partial \varphi_{x}}\right)=\varphi_{t x}+\varphi_{x} \varphi_{x x}+\psi_{x x}=0 \\
& \frac{\delta \hat{\mathcal{L}}}{\delta \psi}=-\frac{\partial}{\partial t}\left(\frac{\partial \hat{\mathcal{L}}}{\partial \psi_{t}}\right)-\frac{\partial}{\partial x}\left(\frac{\partial \hat{\mathcal{L}}}{\partial \psi_{x}}\right)=\frac{\psi_{t x}}{c}+\varphi_{x x}-\chi_{x}=0 \\
& \frac{\delta \hat{\mathcal{L}}}{\delta \chi}=\frac{\partial \hat{\mathcal{L}}}{\partial \chi}-\frac{\partial}{\partial t}\left(\frac{\partial \hat{\mathcal{L}}}{\partial \chi_{t}}\right)-\frac{\partial}{\partial x}\left(\frac{\partial \hat{\mathcal{L}}}{\partial \chi_{x}}\right)=\chi_{t x}+\frac{\chi_{x x}}{c}+\psi_{x}=0 .
\end{aligned}
$$

Equations (9) are thus equivalent to (8). The first equation can be written in the form of conservation law expressed in variables $u$ and $v$ :

$$
u_{t}+\left(u^{2} / 2+v\right)_{x}=0
$$

An equivalent form of (9) in terms of the potentials is :

$$
\begin{aligned}
& \chi_{t}+\frac{\chi_{x}}{c}+\psi=0, \\
& \frac{\psi_{t}}{c}+\varphi_{x}-\chi=0, \\
& \varphi_{t}+\frac{\varphi_{x}^{2}}{2}+\psi=0 .
\end{aligned}
$$

Arbitrary functions depending only on time which appear after the integration (9) with respect to $x$ can always be put vanishing by introducing equivalent potentials.

Due to the Noether theorem, the system admits two additional conservation laws :

$$
\left(\varphi_{x} \frac{\partial \hat{\mathcal{L}}}{\partial \varphi_{t}}+\psi_{x} \frac{\partial \hat{\mathcal{L}}}{\partial \psi_{t}}+\chi_{x} \frac{\partial \hat{\mathcal{L}}}{\partial \chi_{t}}\right)_{t}+\left(\varphi_{x} \frac{\partial \hat{\mathcal{L}}}{\partial \varphi_{x}}+\psi_{x} \frac{\partial \hat{\mathcal{L}}}{\partial \psi_{x}}+\chi_{x} \frac{\partial \hat{\mathcal{L}}}{\partial \chi_{x}}-\hat{\mathcal{L}}\right)_{x}=0
$$

and

$$
\left(\varphi_{t} \frac{\partial \hat{\mathcal{L}}}{\partial \varphi_{t}}+\psi_{t} \frac{\partial \hat{\mathcal{L}}}{\partial \psi_{t}}+\chi_{t} \frac{\partial \hat{\mathcal{L}}}{\partial \chi_{t}}-\hat{\mathcal{L}}\right)_{t}+\left(\varphi_{t} \frac{\partial \hat{\mathcal{L}}}{\partial \varphi_{x}}+\psi_{t} \frac{\partial \hat{\mathcal{L}}}{\partial \psi_{x}}+\chi_{t} \frac{\partial \hat{\mathcal{L}}}{\partial \chi_{x}}\right)_{x}=0
$$

The conservation law (12) corresponds to (5) and can be expressed in variables $u, v$ and $w$ as :

$$
\left(\frac{u^{2}}{2}+\frac{v^{2}}{2 c}+\frac{w^{2}}{2}\right)_{t}+\left(\frac{u^{3}}{3}+u v+\frac{w^{2}}{2 c}\right)_{x}=0 .
$$

Finally, the conservation law (13) corresponding to (6) is :

$$
\begin{aligned}
& \left(\frac{\varphi_{x}^{3}}{6}+\frac{\chi_{x}^{2}}{2 c}+\psi_{x}\left(\varphi_{x}-\chi\right)\right)_{t}- \\
& \quad\left(\frac{\varphi_{t}^{2}}{2}+\frac{\psi_{t}^{2}}{2 c}+\frac{\chi_{t}^{2}}{2}+\varphi_{t}\left(\psi_{x}+\frac{\varphi_{x}^{2}}{2}\right)+\psi_{t}\left(\varphi_{x}-\chi\right)+\frac{\chi_{t} \chi_{x}}{c}\right)_{x}=0 .
\end{aligned}
$$


Using (11b) one obtains : $\varphi_{x}-\chi=-\psi_{t} / c$, and the conservation law (15) can also be expressed in terms of the time and space derivatives of the potentials. However, it cannot be expressed in terms of variables $u, v$ and $w$ and their space and time derivatives.

\section{Properties of the hyperbolic BBM system}

In this section we resume the mathematical properties of the hyperbolic BenjaminBona-Mahony (BBMH) quasilinear system (8).

Theorem

1. For any $c>0$ the system (8) can be rewritten as a symmetric $t$-hyperbolic in the sense of Friedrichs system :

$$
\mathbf{A} \mathbf{U}_{t}+\mathbf{B U}_{x}=\mathbf{C U}, \quad \mathbf{A}=\mathbf{A}^{T}>0, \quad \mathbf{B}=\mathbf{B}^{T}, \quad \mathbf{C}=-\mathbf{C}^{T},
$$

with

$\mathbf{U}=\left(\begin{array}{c}u \\ v \\ w\end{array}\right), \mathbf{A}=\left(\begin{array}{ccc}1 & 0 & 0 \\ 0 & \frac{1}{c} & 0 \\ 0 & 0 & 1\end{array}\right), \mathbf{B}=\left(\begin{array}{ccc}u & 1 & 0 \\ 1 & 0 & 0 \\ 0 & 0 & \frac{1}{c}\end{array}\right), \mathbf{C}=\left(\begin{array}{ccc}0 & 0 & 0 \\ 0 & 0 & 1 \\ 0 & -1 & 0\end{array}\right)$.

The corresponding eigenvalues are given in explicit form :

$$
\lambda^{0}=1 / c, \quad \lambda^{ \pm}=\left(u \pm \sqrt{u^{2}+4 c}\right) / 2, \quad \lambda^{-}<0<\min \left(\lambda^{0}, \lambda^{+}\right) .
$$

The eigenvalues $\lambda^{+}$and $\lambda^{0}$ coincide for $u=1 / c-c^{2}$, i.e. the system is not strictly hyperbolic.

2. It can be rewritten in terms of Riemann invariants :

$$
\begin{array}{ll}
R_{t}^{+}+\lambda^{+} R_{x}^{+}=c w, & R^{+}=v+\int^{u} \lambda^{+}(s) d s, \\
R_{t}^{-}+\lambda^{-} R_{x}^{-}=c w, & R^{-}=v+\int^{u} \lambda^{-}(s) d s, \\
w_{t}+\lambda^{0} w_{x}=-v . &
\end{array}
$$

A closed form expression for the Riemann invariants $R^{ \pm}$is :

$$
R^{ \pm}=v+\frac{u^{2}}{4} \pm c\left(z \sqrt{1+z^{2}}+\ln \left(z+\sqrt{1+z^{2}}\right)\right), \quad z=\frac{u}{2 \sqrt{c}} .
$$

3. The fields $\lambda^{ \pm}$and $\lambda^{0}$ are genuinely non-linear and linearly degenerate, respectively, in the sense of Lax [36].

The proof of the first two statements is direct. To prove the third statement, it is sufficient to find the derivatives $\frac{\partial \lambda^{ \pm}}{\partial R^{ \pm}}$and show that they cannot vanish. Let us remark first that $u$ is implicitly defined from

$$
R^{+}-R^{-}=\int^{u}\left(\lambda^{+}(s)-\lambda^{-}(s)\right) d s=\int^{u} \sqrt{s^{2}+4 c} d s .
$$




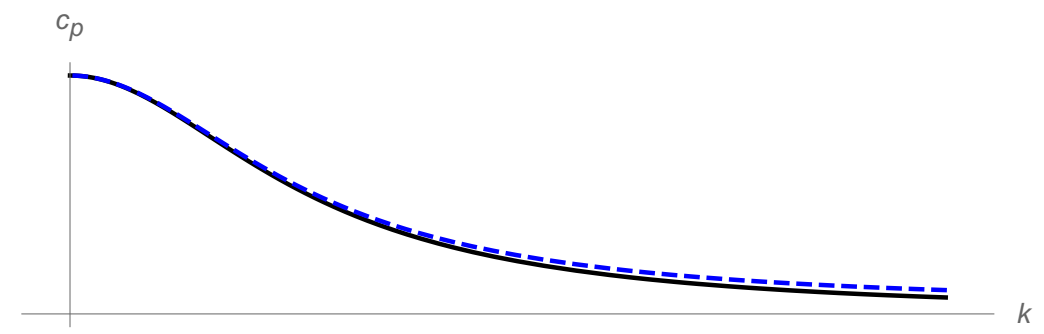

Figure 1: Dispersion curve for the BBM equation (continuous line) and for its hyperbolic version (dashed line). The error between the corresponding phase velocities is $\mathcal{O}(1 / c)$ uniformly relative to $k$.

In particular, it implies

Hence,

$$
\frac{\partial \lambda^{+}}{\partial R^{+}}=\frac{d \lambda^{+}}{d u} \frac{\partial u}{\partial R^{+}}=\frac{1}{2}\left(1+\frac{u}{\sqrt{u^{2}+4 c}}\right) \frac{1}{\sqrt{u^{2}+4 c}}>0 .
$$

Analogous proof can be done for the eigenvalue $\lambda^{-}$. The degeneracy of the eigenfield $\lambda^{0}$ is obvious.

\section{Dispersion relation}

Linearizing the equations (8) on the state $u=u_{0}, v=0, w=0$, one obtains the system for perturbations (denoted below by the same letters $u, v$ and $w$ ):

$$
u_{t}+u_{0} u_{x}+v_{x}=0, \quad \frac{v_{t}}{c}+u_{x}=w, \quad w_{t}+\frac{w_{x}}{c}=-v .
$$

Looking for the monochromatic waves satisfying (17) which are proportional to $\exp (i(k x-\omega t))$, with $k$ and $\omega$ being the wave number and frequency, one obtains the dispersion relation between the phase velocity $c_{p}=\omega / k$ and wave number $k$ :

$$
\left(c_{p}\left(c_{p}-u_{0}\right)\left(c_{p}-\frac{1}{c}\right)+1\right) \frac{k^{2}}{1+k^{2}}=c\left(c_{p}-\frac{u_{0}}{1+k^{2}}\right) .
$$

It can also be written as :

$$
\frac{c_{p}}{c}=\frac{1}{k^{2}\left(c_{p}-1 / c\right)}+\frac{1}{c_{p}-u_{0}} .
$$

Consider the case $u_{0}>1 / c>0$. For any $k$ there are three roots $c_{p}$ of (18). The root $c_{p}$ belonging to the interval $\left(1 / c, u_{0}\right)$ tends to the phase velocity of the BBM equation (2) as $c \rightarrow \infty$ (see Figure 1). The corresponding error 
$\mathcal{O}(1 / c)$ is uniform with respect to $k$. It is worth to note that the phase velocity defined by (18) is larger than the phase velocity (2) of the exact BBM equation. This property is well known for hyperbolic systems with relaxation as the subcharacteristic condition (or Whitham's condition) : the 'frozen' sound speed (i.e. the sound speed of non-relaxed system) is larger then the 'equilibrium' sound speed (the sound speed of relaxed system) [46]. This property is thus also valid for our hyperbolic approximation of the BBM equation.

\section{Travelling wave solutions of the BBM equa- tion}

The travelling wave solutions of the BBM equation $u=u(\xi), \xi=x-D t$ satisfy the equation :

$$
-D\left(u-u^{\prime \prime}\right)+\frac{u^{2}}{2}=c_{1}, \quad c_{1}=\text { const } .
$$

Here 'prime' means the derivative with respect to $\xi$. It implies the first integral

$$
D \frac{u^{\prime 2}}{2}=-\frac{u^{3}}{6}+D \frac{u^{2}}{2}+c_{1} u+c_{2}=\frac{1}{6}\left(u-u_{1}\right)\left(u-u_{2}\right)\left(u_{3}-u\right), \quad c_{2}=\text { const },
$$

where new constants $u_{1} \leq u_{2} \leq u_{3}$ are introduced. They related with $D, c_{1}$ and $c_{2}$ :

$$
D=\frac{1}{3}\left(u_{1}+u_{2}+u_{3}\right), \quad c_{1}=-\frac{1}{6}\left(u_{1} u_{2}+u_{1} u_{3}+u_{2} u_{3}\right), \quad c_{2}=\frac{1}{6} u_{1} u_{2} u_{3} .
$$

In the following, we will consider only positive solutions $\left(0<u_{1}<u_{2}<u<u_{3}\right)$ (the negative solutions can be recovered by the symmetry $u \rightarrow-u$ and $D \rightarrow$ $-D)$. The periodic solution $u(x)$ is given by :

$$
u(x)=u_{2}+a \mathrm{cn}^{2}(\eta, m),
$$

where

$$
m=\frac{u_{3}-u_{2}}{u_{3}-u_{1}}, \quad a=u_{3}-u_{2}, \quad \eta=\frac{\xi+\xi_{0}}{2 \sqrt{3 D}} \sqrt{\frac{a}{m}}, \quad \xi_{0}=\text { const } .
$$

Here $\operatorname{cn}(\eta, m)=\cos (\varphi(\eta, m))$, where $\varphi$ is defined implicitly from

$$
\eta=\int_{0}^{\varphi(\eta, m)} \frac{d \theta}{\sqrt{1-m \sin ^{2} \theta}} .
$$

The solitary wave solution obtained for the values $u_{1}=u_{2}>0, a=u_{3}-u_{2}$ is in the form

$$
u(\xi)=u_{2}+\frac{a}{\cosh ^{2}(\eta)}, \eta=\frac{\xi+\xi_{0}}{2 \sqrt{1+\frac{3 u_{2}}{a}}}, \xi=x-D t, D=u_{2}+\frac{a}{3}, \xi_{0}=\text { const } .
$$




\section{Travelling wave solutions for the hyperbolic BBM equation}

Traveling wave solutions of the hyperbolic equations (8) are of the form : $(u, v, w)=(u, v, w)(\xi)$, where $\xi=x-D t$ and $D=$ const is the wave velocity. A before, we denote by 'prime' the derivative with respect to $\xi$. From the conservation laws (10) and (14), and the last equation of (8) we have :

$$
\begin{aligned}
-D u+u^{2} / 2+v & =c_{1}=\text { const }, \\
-D\left(\frac{u^{2}}{2}+\frac{v^{2}}{2 c}+\frac{w^{2}}{2}\right) & +\left(\frac{u^{3}}{3}+u v+\frac{w^{2}}{2 c}\right)=-c_{2}=\text { const }, \\
v & =\left(D-\frac{1}{c}\right) w^{\prime} .
\end{aligned}
$$

Here $c_{i}$ are constants. As in the case of the BBM equation, consider the polynomial

$$
P(u)=-u^{3}+3 D u^{2}+6 c_{1} u+6 c_{2}=\left(u-u_{1}\right)\left(u-u_{2}\right)\left(u_{3}-u\right) .
$$

For simplification purposes, we keep the same notations as in the case of exact $\mathrm{BBM}$ equation, even if the solution depends now on $c$. One finally has :

$$
\begin{aligned}
& D\left(D-\frac{1}{c}\right)^{2} \frac{w^{\prime 2}}{2 c}+\left(D-\frac{1}{c}\right) \frac{w^{2}}{2}=\frac{1}{6} P(u), \\
& v=\left(D-\frac{1}{c}\right) w^{\prime}=\frac{1}{6} \frac{d P}{d u} .
\end{aligned}
$$

Differentiating (26a) with respect to $\xi$, one obtains

$$
D\left(D-\frac{1}{c}\right)^{2} \frac{w^{\prime} w^{\prime \prime}}{c}+\left(D-\frac{1}{c}\right) w w^{\prime}=\frac{1}{6} \frac{d P}{d u} u^{\prime} .
$$

Thus, the system (26a)- (26b) has the following form

$$
\left(D-\frac{1}{c}\right) w^{\prime}=\frac{1}{6} \frac{d P}{d u}, \quad D\left(D-\frac{1}{c}\right) \frac{w^{\prime \prime}}{c}=u^{\prime}-w .
$$

Differentiating the first equation again with respect to $\xi$, and eliminating $w^{\prime \prime}$, one obtains the final system of ordinary differential equation (ODE) which is suitable for the numerical construction of the travelling wave solutions :

$$
u^{\prime}=\frac{w}{1-\frac{D}{6 c} \frac{d^{2} P}{d u^{2}}}, \quad w^{\prime \prime}=\frac{1}{6} \frac{d^{2} P}{d u^{2}} \frac{w}{\left(D-\frac{1}{c}\right)\left(1-\frac{D}{6 c} \frac{d^{2} P}{d u^{2}}\right)} .
$$

Since $\frac{d^{2} P}{d u^{2}}=6(D-u), D=\left(u_{1}+u_{2}+u_{3}\right) / 3$, one finally has

$$
u^{\prime}=\frac{w}{1-\frac{D(D-u)}{c}}, \quad w^{\prime \prime}=\frac{w(D-u)}{\left(D-\frac{1}{c}\right)\left(1-\frac{D(D-u)}{c}\right)} .
$$



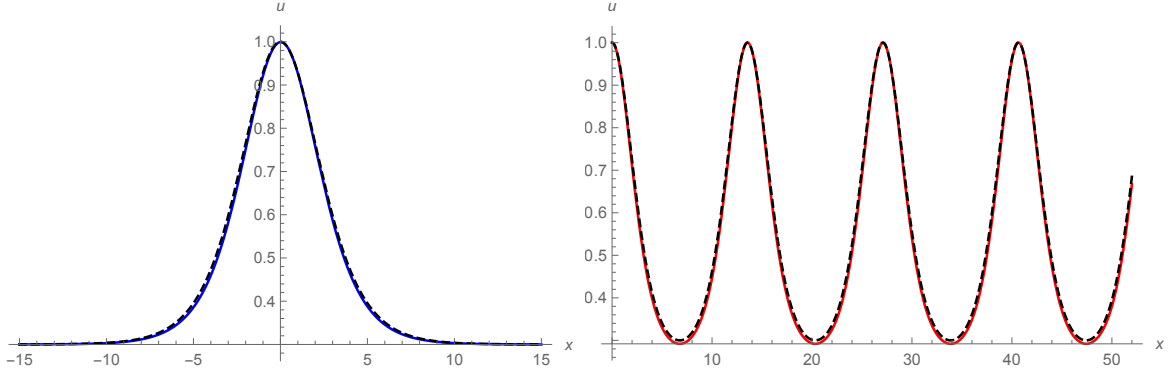

Figure 2: Left figure : the exact solitary wave solution of the BBM equation (25) with parameters $u_{1}=u_{2}=0.3, u_{3}=1$ (dashed line) and the solution of the hyperbolic BBM system for $c=30$ (continuous line) are shown. Right figure : periodic solution of the BBM equation (22) with parameters $u_{1}=0.2, u_{2}=$ $0.3, u_{3}=1$ (dashed line) is approximated by the solution of the hyperbolic BBM system with $c=100$. In both cases, the accuracy of the approximation is very good.

If $c \rightarrow \infty$, one has $w \rightarrow u^{\prime}$, and we recover the travelling wave solutions of the BBM equation. Unfortunately, we could not find the closed form analytical solutions of this ODE system even in the limit case of solitary waves $u_{1}=u_{2}<$ $u_{3}$. Nevertheless, one can show that the accuracy of the hyperbolic system is very good. In Figure 2 the comparison of solutions is made for $c=30$ in the case of solitary wave (25) having the parameters $u_{1}=u_{2}=0.3, a=u_{3}-u_{2}=0.7$. For the numerical solution of (27), we took the initial data at $\xi=\xi_{0}<0$ in the following way :

$$
w\left(\xi_{0}\right)=\epsilon, w^{\prime}\left(\xi_{0}\right)=\lambda \epsilon, u\left(\xi_{0}\right)=u_{2}+\frac{\epsilon}{\lambda\left(1-D\left(D-u_{2}\right) / c\right)},
$$

where

$$
\lambda=\sqrt{\frac{D-u_{2}}{\left(D-\frac{1}{c}\right)\left(1-\frac{D\left(D-u_{2}\right)}{c}\right)}} .
$$

For $\xi_{0}=-20$ one takes $\epsilon \approx 0.00000215$ to have the maximum of the solitary wave at $\xi=0$. To approximate the periodic solution of the BBM equation (22) with parameters $u_{1}=0.2, u_{2}=0.3, u_{3}=1$ (dashed line on the right Figure 2 ), we take $c=100$ and the initial data $u(0)=u_{3}, w(0)=0, w^{\prime}(0) \approx-0.20005$. In both cases, the accuracy of the approximation is very good. 


\section{Comparison of non-stationary solutions}

\subsection{Initial conditions}

To compare non-stationary solutions, we need first to understand how to form an initial condition for the hyperbolic BBM system from an initial condition for the BBM equation. In the case of a smooth initial condition $u(0, x)$, one has to take $w(0, x)=\approx u_{x}(0, x)$. How to find $v(0, x)$ ? One has $v(0, x) \approx-u_{t x}(0, x)$. So, the second derivative $u_{t x}(0, x)$ should be found. The BBM equation can be written as :

$$
\mathcal{K}_{t}=-u u_{x}, \quad \mathcal{K}=u-u_{x x}=T[u] .
$$

The operator $T$ has its inverse given explicitly as :

$$
u(t, x)=T^{-1}[\mathcal{K}](t, x)=\frac{1}{2} \int_{-\infty}^{\infty} e^{-|x-y|} \mathcal{K}(t, y) d y .
$$

Differentiating $u(t, x)$ with respect to $t$, one obtains :

$$
\begin{aligned}
u_{t}(t, x) & =\frac{1}{2} \int_{-\infty}^{\infty} e^{-|x-y|} \mathcal{K}_{t}(t, y) d y \\
& =-\frac{1}{2} \int_{-\infty}^{\infty} e^{-|x-y|}\left(\frac{u^{2}}{2}\right)_{y} d y \\
& =\frac{1}{4} \int_{-\infty}^{\infty} u^{2} e^{-|x-y|} \operatorname{sgn}(x-y) d y \\
& =\frac{1}{4} \int_{-\infty}^{x} u^{2} e^{-(x-y)} d y-\frac{1}{4} \int_{x}^{+\infty} u^{2} e^{(x-y)} d y \\
& =\frac{1}{4} e^{-x} \int_{-\infty}^{x} u^{2} e^{y} d y-\frac{1}{4} e^{x} \int_{x}^{+\infty} u^{2} e^{-y} d y .
\end{aligned}
$$

Finally, for $u_{t x}$ one has :

$$
\begin{aligned}
u_{t x}(t, x) & =-\frac{1}{4} e^{-x} \int_{-\infty}^{x} u^{2} e^{y} d y-\frac{1}{4} e^{x} \int_{x}^{+\infty} u^{2} e^{-y} d y+\frac{u^{2}(t, x)}{2} \\
& =\frac{u^{2}(t, x)}{2}-\frac{1}{4} \int_{-\infty}^{+\infty} e^{-|x-y|} u^{2}(t, y) d y
\end{aligned}
$$

So, we take

$$
v(0, x)=-u_{t x}(0, x)=\frac{1}{4} \int_{-\infty}^{+\infty} e^{-|x-y|} u^{2}(0, y) d y-\frac{u^{2}(0, x)}{2} .
$$

\subsection{Numerical solutions}

For the tests below, we take the initial data $u(0, x)$ having the property $\int_{-\infty}^{+\infty} u(0, x) d x=$ $2 L$. The parameter $L$ having the meaning of 'total mass' will vary. A Godunovtype method is used in all the computations with a uniform mesh size $\Delta x_{i}=0.05$ 
on a domain of size $x \in[-300,300]$, and a time step $\Delta t$ determined from the Courant-Friedrich-Lewy (CFL) condition for the stability of the hyperbolic solver, see Appendix A for more detail. The Neumann boundary condition was employed on the left and right of the boundaries during the computations.

\subsubsection{Test 1}

For the hyperbolic BBM system, we initially take $u=1$ on the interval $[-L, L]$, and $u=0$ outside this interval. The function $v(0, x)$ is computed from (31) that gives the explicit form:

$$
v(0, x)= \begin{cases}\frac{1}{4}(\exp (x+L)-\exp (x-L)), & x<-L, \\ -\frac{1}{4}(\exp (-x-L)+\exp (x-L)), & x \in[-L, L], \\ \frac{1}{4}(\exp (-x+L)-\exp (-x-L)), & x>L .\end{cases}
$$

It is also discontinuous at $x= \pm L$. The function $w(0, x)$ is taken zero everywhere. For the BBM equation, the initial discontinuous data are replaced by the hyperbolic tangent :

$$
u(0, x)= \begin{cases}\frac{1}{2}\left(1+\tanh \left(\frac{x+L}{\alpha}\right)\right), & x \leq 0, \\ \frac{1}{2}\left(1-\tanh \left(\frac{x-L}{\alpha}\right)\right), & x>0,\end{cases}
$$

where $\alpha$ is a positive constant, and is taken to be $\alpha=2$ here.

Figure 3 shows numerical results for $L=50$ and $L=100$ at time $t=200$, where the parameter value $c=30$ was used in the solver for the hyperbolic BBM system in both cases. The solutions of the BBM equation and of the hyperbolic BBM system are in good qualitative agreement.

To show the convergence of the hyperbolic BBM solution to the BBM one, we perform a parameter study on $c$ for $c=30,50$, and 100 . The results are presented in Figure 4 at time $t=200$ in the case of $L=100$.

\subsubsection{Test 2}

We take the Gaussian profile :

$$
u(0, x)=\frac{2}{\sqrt{\pi}} \exp \left(-x^{2} / L^{2}\right) .
$$

The function $w(0, x)$ is the derivative of $u(0, x)$ :

$$
w(0, x)=-\frac{4 x}{L^{2} \sqrt{\pi}} \exp \left(-x^{2} / L^{2}\right) .
$$

The expression for $v(0, x)$ is found from (31):

$$
\begin{gathered}
v(0, x)=-\frac{2}{\pi} \exp \left(-2 x^{2} / L^{2}\right)+\frac{L \exp \left(L^{2} / 8-x\right)}{2 \sqrt{2 \pi}}\left(1-\operatorname{erf}\left(\frac{L^{2}-4 x}{2 \sqrt{2} L}\right)\right)+ \\
\frac{L \exp \left(L^{2} / 8+x\right)}{2 \sqrt{2 \pi}}\left(1-\operatorname{erf}\left(\frac{L^{2}+4 x}{2 \sqrt{2} L}\right)\right),
\end{gathered}
$$



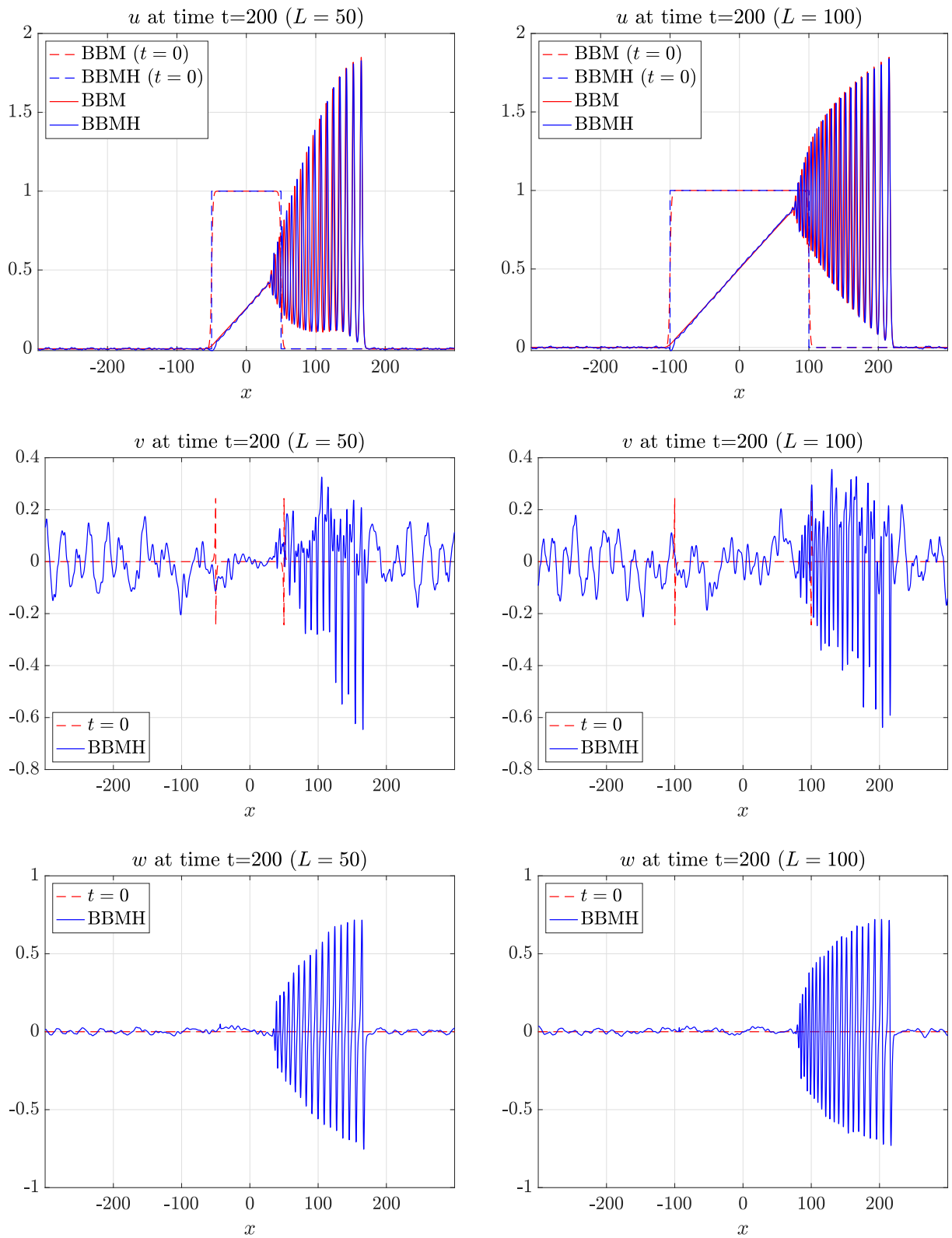

Figure 3: Numerical results for test case 1 with the exact initial condition (32) for $v(0, x)$; snapshot of solutions are shown at time $t=200$ for $L=50$ and $L=100$. In both cases, parameter value $c=30$ was used for the hyperbolic BBM (BBMH) system. 


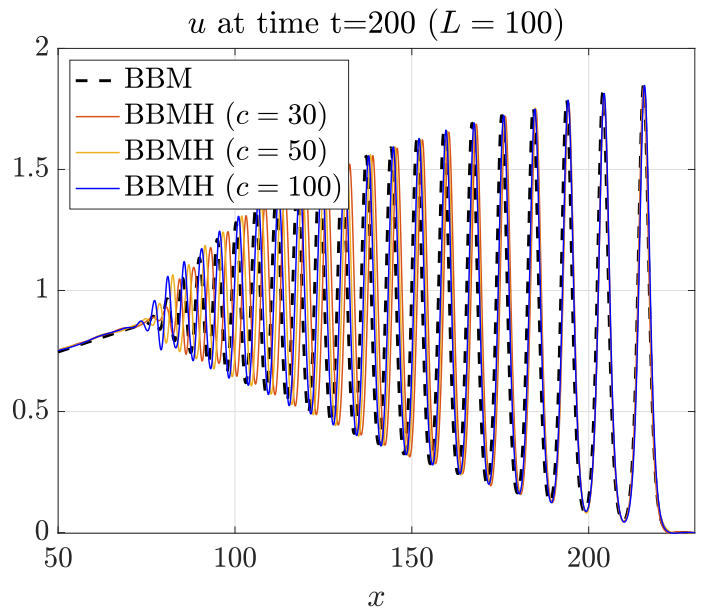

Figure 4: A convergence study of the solution on $c$ for test case 1. Solutions are shown for $c=30,50$, and 100 in the case of $L=100$ at time $t=200$; only the partial solutions in the region $x \in[50,230]$ are drawn.

where

$$
\operatorname{erf}(z)=\frac{2}{\sqrt{\pi}} \int_{0}^{z} \exp \left(-y^{2}\right) d y .
$$

Note that the function $v(0, x)$ given by $(33)$ attains its minimum at $x=0$. This value is

$$
v_{m}(L)=-\frac{2}{\pi}+\frac{L \exp \left(L^{2} / 8\right)}{\sqrt{2 \pi}}\left(1-\operatorname{erf}\left(\frac{L}{2 \sqrt{2}}\right)\right) .
$$

The asymptotic formula for large $L$ is :

$$
v_{m}(L) \approx-\frac{8}{\pi L^{2}} .
$$

Thus, for large $L$ we can take $v(0, x) \approx 0$.

The corresponding numerical results are shown in Fig. 5 in the case of $L=20$ and $c=30$, where we have employed two different initial condition for $v$ in the computation : $v(0, x)$ is given either by $(33)$ or $v(0, x)=0$. No significant differences were observed between the solutions in this respect. Figure 6 shows the solution behavior $t=200$ for different values of $L: L=50$ and $L=100$. In both cases we took $c=30$ and $v(0, x)=0$. As in the Test 1 , to show the convergence of the 'hyperbolic' BBM solution to the BBM one, we perform a parameter study on $c$ for $c=30,50$, and 100 ( see Figure 7 for the solutions at time $t=200$ in the case of $L=100$ ). 

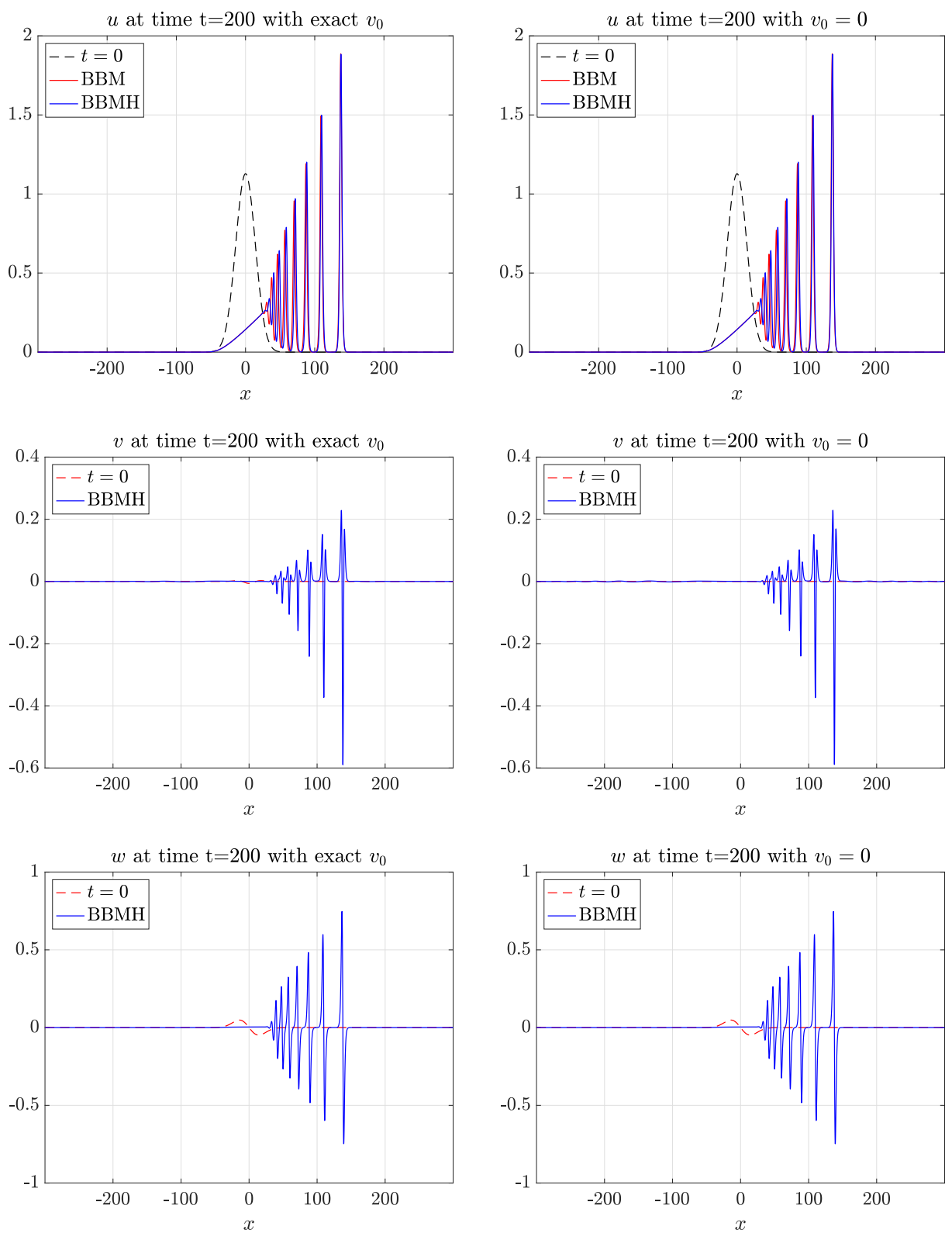

Figure 5: Numerical results for the test case 2 with two different initial conditions for $v$. Snapshot solutions are shown at time $t=200$ for the case of $L=20$ and $c=30$. 

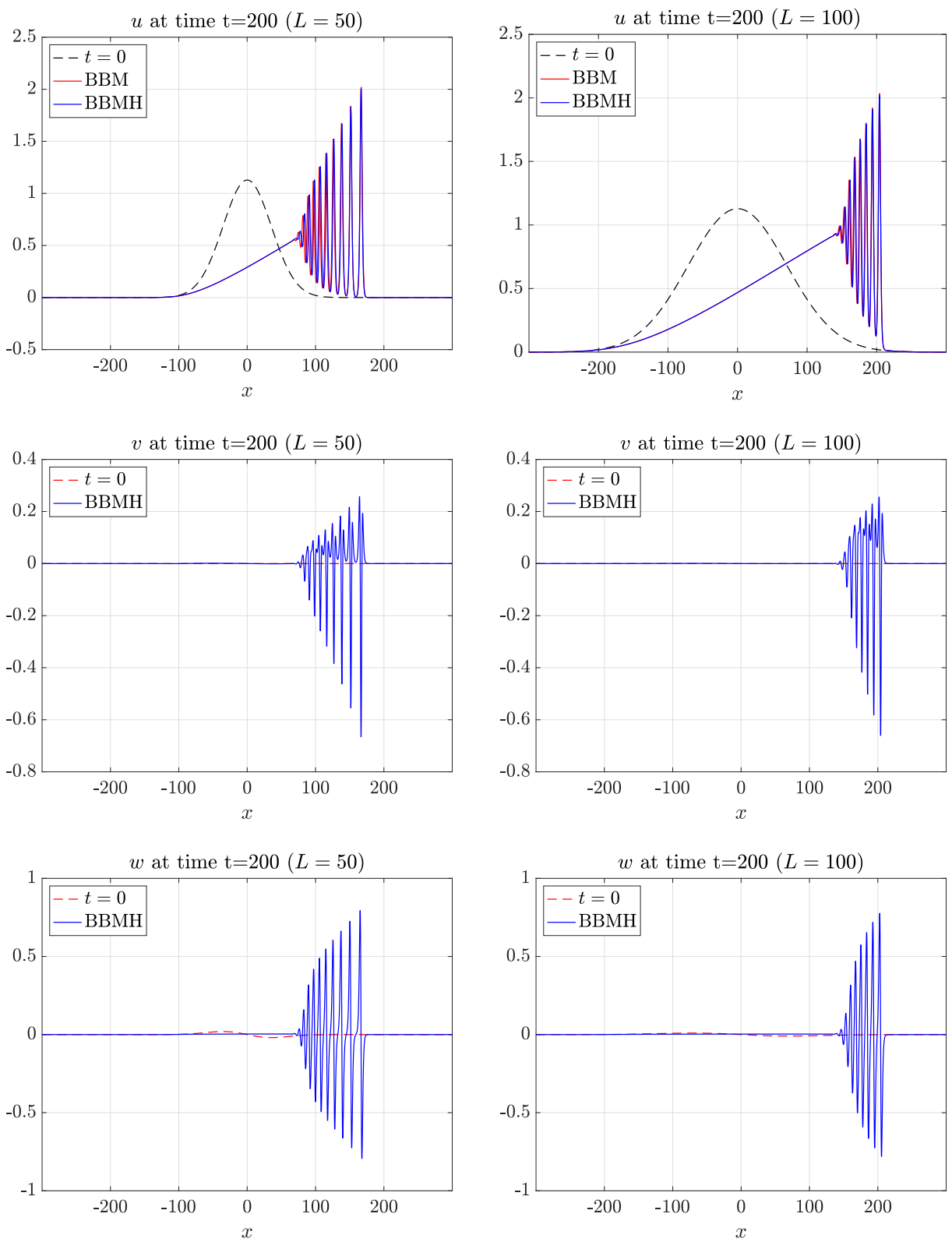

Figure 6: Numerical results for test case 2 with $v_{0}=0$; snapshot of solutions are shown at time $t=200$ for $L=50$ and $L=100$. Parameter value $c=30$ was used in both cases. 


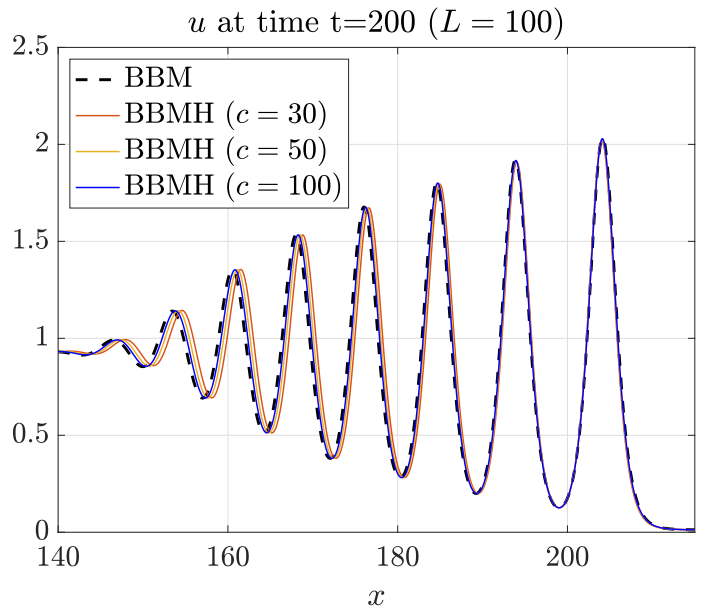

Figure 7: A convergence study of the solution on $c$ for the test case 2. Solutions are shown for $c=30,50$, and 100 in the case of $L=100$ at time 200; only the partial solutions in the region $x \in[140,215]$ are shown.

\section{Conclusion}

A hyperbolic approximation (8) of the BBM equation based on an 'augmented Lagrangian' formulation is proposed. The corresponding equations can be written as a symmetric $t$-hyperbolic in the sense of Friedrichs system. It allows us to use the state-of-the-art numerical methods for hyperbolic conservation laws for solving the 'hyperbolized' BBM system. Recently, there has been an increasing interest to the Riemann problem for the dispersive equations $[28,29,19,18,20,21,12,22,24,25]$. The approximation we proposed could be a new numerical tool for solving the Cauchy problem for the BBM equation with discontinuous initial data.

Acknowledgments SG thanks François Dubois for helpful discussion. KMS was partially supported by the Grant MOST 109-2115-M-002-012.

\section{A Numerical method}

\section{A.1 BBM equation}

To find approximate solutions to BBM equation, we use the hyperbolic-elliptic splitting approach developed previously in [39, 24]. This algorithm consists of two steps. In the first step, the hyperbolic step, we employ the state-of-theart method for hyperbolic conservation laws for the numerical resolution of the equation

$$
\mathcal{K}_{t}+\left(\frac{u^{2}}{2}\right)_{x}=0, \quad \text { with } \quad \mathcal{K}=u-u_{x x}
$$


over a time step $\Delta t$. In the second step, the elliptic step, using the approximate solution $\mathcal{K}$ computed during the hyperbolic step, we invert numerically the elliptic operator:

$$
u-u_{x x}=\mathcal{K}
$$

with prescribed boundary conditions based on a fourth-order compact scheme [37].

More precisely, in the hyperbolic step, we use the semi-discrete finite volume method written in a wave-propagation form as before [24], but employ a different solution reconstruction technique, the BVD (boundary variation diminishing) principle, which is more robust than the classical one for the interpolated states ( $\mathcal{K}$ for BBM) at cell boundaries (cf. [13] and the references cited therein). These reconstructed variables form the basis for the initial data of the Riemann problems, where the solutions of the Riemann problems (obtained from the local Lax-Friedrichs approximate solver [38] for BBM) are then used to construct the fluctuations in the spatial discretization that gives the right-hand side of the system of ODEs (cf. $[33,34]$ ). To integrate the ODE system in time, the strong stability-preserving (SSP) multistage Runge-Kutta scheme [30, 45] is used. In particular, for the numerical results presented in this paper, the third-order SSP scheme was employed together with the pair of third- and fifth-order WENO (weighted essentially non-oscillatory) scheme in the BVD reconstruction process.

\section{A.2 Hyperbolic BBM system}

As in [23], we use a fractional-step approach for the numerical resolution of the hyperbolic BBM system : at each time step we alternate between by solving the homogeneous (hyperbolic) part of the symmetric $t$-hyperbolic system (16a)

$$
\left[\begin{array}{c}
u \\
v / c \\
w
\end{array}\right]_{t}+\left[\begin{array}{c}
u^{2} / 2+v \\
u \\
w / c
\end{array}\right]_{x}=0
$$

over a time step $\Delta t$, and the ODEs

$$
\left[\begin{array}{c}
u \\
v / c \\
w
\end{array}\right]_{t}=\left[\begin{array}{c}
0 \\
w \\
-v
\end{array}\right]
$$

using the inital data from the previous step and the same time step. Here the numerical method we employed for (34a) is the same as for the BBM equation (see A.1, hyperbolic step), and we use the exact solution of (34b) for the update of the solution in the ODE step :

$$
\begin{aligned}
& u=u_{0}, \\
& v=v_{0} \cos (\sqrt{c} t)+w_{0} \sqrt{c} \sin (\sqrt{c} t), \\
& w=-\frac{v_{0}}{\sqrt{c}} \sin (\sqrt{c} t)+w_{0} \cos (\sqrt{c} t),
\end{aligned}
$$

where $u_{0}, v_{0}$ and $w_{0}$ is the solution of the homogeneous system (34a). Such a splitting procedure was also used in [23] in the case of the SGN equations. 


\section{References}

[1] Antuono M, Liapidevskii V and Brocchini M 2009 Dispersive Nonlinear Shallow-Water Equations, Studies in Applied Mathematics 122(1) 1-28.

[2] Antoine X, Arnold A, Besse C, Ehrhardt M and Schädle A 2008 A review of transparent and artificial boundary conditions techniques for linear and nonlinear Schrdinger equations, Commun. Comput. Phys. 4 729-796.

[3] Arnold A, Ehrhardt M and Sofronov I 2003 Discrete transparent boundary conditions for the Schrödinger equation: Fast calculation, approximation, and stability. Commun. Math. Sci. 3 501-556.

[4] Bassi C, Bonaventura L, Busto S and Dumbser M 2020 A hyperbolic reformulation of the Serre-Green-Naghdi model for general bottom topographies, Computers and Fluids 2012104716

[5] Benjamin T B, Bona J L and Mahony J J 1972 Model equations for long waves in nonlinear dspersive systems. Phil. Trans. Royal Soc. A 272 47-78.

[6] Besse C, Ehrhardt M and I. Lacroix-Violet I 2016 Discrete Artificial boundary conditions for the linearized Korteweg-de Vries equation, Num.Meth. for PDE, V. 32, Issue 5, 1455-1484.

[7] Besse C, Mesognon B and Noble P (2016) Discrete Artificial Boundary Condition for the Benjamin-Bona-Mahoney equation, Preprint 2016, hal01305360.

[8] Bourgeois S, Favrie N and Lombard B 2020 Dynamics of a regularized and bistable Ericksen bar using an extended Lagrangian approach, hal.archivesouvertes.fr/hal-02519362

[9] Busto S, Dumbser M, Escalante C, Favrie N and Gavrilyuk S 2021 On high order ADER discontinuous Galerkin schemes for first order hyperbolic reformulations of nonlinear dispersive systems, J. Sci. Comput, 8748 .

[10] Cattaneo C 1958 Sur une forme de l'équation de la chaleur éliminant le paradoxe d'une propagation instantanée, Comptes Rendues de l'Académie des Sciences 247, 431-433.

[11] Chesnokov A A and Liapidevskii V Y 2020 Hyperbolic model of internal solitary waves in a three layer stratified fluid, European Phys. J. Plus 135 590.

[12] Congy T, El G A, Hoeffer M A and Shearer M 2021 Dispersive Riemann problem for the Benjamin-Bona-Mahony equation https://arxiv.org/abs/2012.14579.

[13] Deng X, Inaba S, Xie B, Shyue K M and Xiao F 2018 High fidelity discontinuity-resolving reconstruction for compressible multiphase flows with moving interfaces, J. Comput. Phys. 371 945-966.

[14] Dhaouadi F, Favrie S and Gavrilyuk S 2018 Extended Lagrangian approach for the defocusing nonlinear Schrödinger equation, Studies in Applied Mathematics 142 (3), 336-358.

[15] Duchene 2019, Rigorous justification of the Favrie-Gavrilyuk approximation to the Serre-Green-Naghdi model, Nonlinearity 32, 3772-3797. 
[16] Dumbser M, Peshkov I, Romenski E and Zanotti O 2016 High order ADER schemes for a unified first order hyperbolic formulation of continuum mechanics: Viscous heat-conducting fluids and elastic solids, Journal Comp. Physics 314 824-862.

[17] Dumbser M, Peshkov I, Romenski E and Zanotti O 2017 High order ADER schemes for a unified first order hyperbolic formulation of Newtonian continuum mechanics coupled with electro-dynamics, Journal Comp. Physics $348298-342$.

[18] Gurevich A V, Krylov A V and El G A 1990 Nonlinear modulated waves in dispersive hydrodynamics, J. Exp. Theor. Phys. 98 1605-1626.

[19] El G A, Geogjaev V V, Gurevich A V and Krylov A V 1995 Decay of an initial discontinuity in the defocusing NLS hydrodynamics, Physica D: Nonlinear Phenomena 87 186-192.

[20] El G A, Grimshaw R H J and Smyth N F 2006 Unsteady undular bores in fully nonlinear shallow-water theory, Phys. Fluids 18027104.

[21] El G A and Hoefer M 2016 Dispersive shock waves and modulation theory, Physica D 333 11-65.

[22] El G A, Hoefer M A and Shearer M 2016 Expansion shock waves in regularized shallow water theory, Proc. Royal Soc. A 472.

[23] Favrie N and Gavrilyuk S 2017 A rapid numerical method for solving SerreGreen-Naghdi equations describing long free surface gravity waves, Nonlinearity 30 (7).

[24] Gavrilyuk S, Nkonga B, Shyue K M and Truskinovsky L 2020 Stationary shock-like transition fronts in dispersive systems, Nonlinearity 33 54775509 .

[25] Gavrilyuk S and Shyue K M 2021 Singular solutions of the BBM equation : analytical and numerical study, https://hal.archives-ouvertes.fr/hal03220465/document.

[26] Green A E, Laws N and Naghdi P M 1974 On the theory of water waves, Proc. R. Soc. Lond. A 338 43-55.

[27] Green A E and Naghdi P M 1976 A derivation of equations for wave propagation in water of variable depth, J. Fluid Mech. 78 237-246.

[28] Gurevich A, Pitaevskii L 1974 Nonstationary structure of a collisionless shock wave, JETP 38291 - 297.

[29] Gurevich A V and Krylov A V 1987 Dissipationless shock waves in media with positive dispersion, Zh. Eksp. Teor. Fiz. 92 1684-1699.

[30] Gottlieb S, Shu C W and Tadmor E 2001 Strong stability-preserving highorder time discretization methods, SIAM Review 43 89-112.

[31] Kamchatnov A M 2000 Nonlinear Periodic Waves and Their Modulations: An Introductory Course, World Scinetific Publishing.

[32] Kazakova M and Noble P 2020 Discrete Transparent Boundary Conditions for the Linearized Green-Naghdi System of Equations, SIAM J. Numer. Anal., 58(1), 657-683. 
[33] Ketcheson D I and LeVeque R J 2008 WENOCLAW: A higher order wave propagation method, in: Hyperbolic Problems: Theory, Numerics, Applications, Springer-Verlag, 609-616.

[34] Ketcheson D I, Parsani M and LeVeque R J 2013 High-order wave propagation algorithm for hyperbolic systems, SIAM J. Sci. Comput. 35(1) A351-A377.

[35] Lannes D and Weynans L 2020 Generating boundary conditions for a Boussinesq system, Nonlinearity 33 6868-6889.

[36] Lax P D 19572 Hyperbolic systems of conservation laws, II., Comm. Pure Appl. Math. 19 537-566.

[37] Lele S K 1992 Compact finite difference schemes with spectral-like resolution, J. Comput. Phys. 103 16-43.

[38] LeVeque, R J Finite Volume Methods for Hyperbolic Problems, Cambridge University Press, 2002.

[39] Le Métayer O, Gavrilyuk S and Hank S 2010 A numerical scheme for the Green-Naghdi model, J. Comp. Phys. 229 2034-2045.

[40] Liapidevskii V Yu and Gavrilova K N 2008 Dispersion and blockage effects in the flow over a sill, J. Appl. Mech. Tech. Physics bf 49 (1) 34-45.

[41] Olver P J 1979 Euler operators and conservation laws of the BBM equation, Math. Proc. Camb. Phil. Soc. 85143 - 160.

[42] Olver P J 1980 On the Hamiltonian structure of evolution equations, Math. Proc. Camb. Phil. Soc. $8871-88$.

[43] Richard G 2021 An extension of the Boussinesq-type models to weakly compressible flows, European J. Mechanics - B/Fluids (accepted).

[44] Serre F 1953 Contribution à l'étude des écoulements permanents et variables dans les canaux, La Houille Blanche 8 374-388.

[45] Shu C W 2009 High order weighted essentially nonoscillatory schemes for convection dominated problems, SIAM Review 5 82-126.

[46] Whitham G B 1974 Linear and Nonlinear Waves, John Wiley and Sons. 